\title{
Inaccuracy of accumulated degree day models for estimating terrestrial post-mortem intervals in Cape Town, South Africa
}

Marisa N.S. Forbes ${ }^{\mathrm{a}, \mathrm{b}}$

Marisa.Forbes@unb.ca

Devin A. Finaughty ${ }^{\mathrm{b}, *}$

devfinaughty@hotmail.com, devin.finaughty@uct.ac.za

\section{Kelly L.}

k.miles@unb.ca

Victoria E. G

Victoria.Gibbon@uct.ac.za

${ }^{a}$ Department of Biology, University of New Brunswick, Fredericton, N.B., Canada

${ }^{\mathbf{b}}$ Department of Human Biology, University of Cape Town, Cape Town, W.C., South Africa

${ }^{*}$ Corresponding author at: 5 Iry Lane, TokaiRm. 3.11, Anatomy Building, Faculty of Health Sciences, University of Cape Town, Anzio Road, Observatory, (794225), Cape Town, South Africa.

Abstract

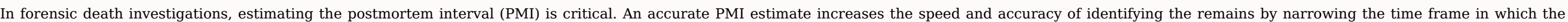

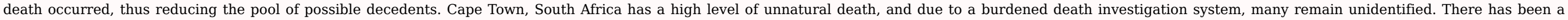

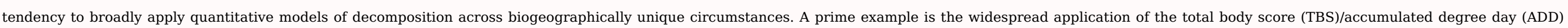

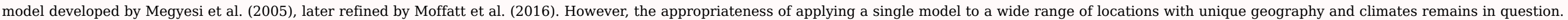

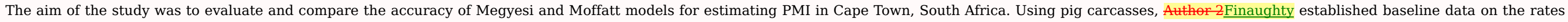

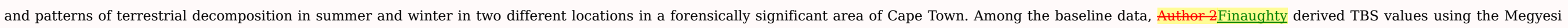

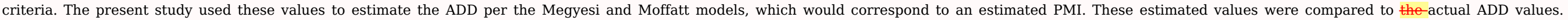

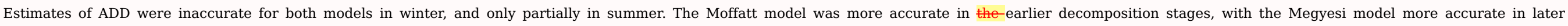

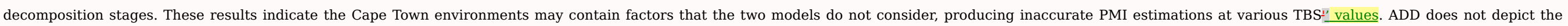

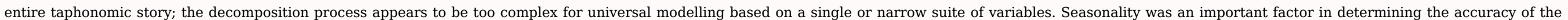

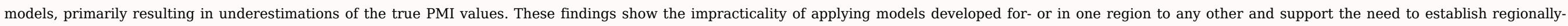

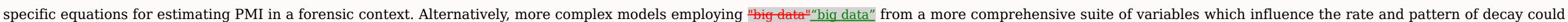
be developed.

Keywords: Accumulated degree days; Decomposition; Total body score; Forensic taphonomy; Postmortem interval

\section{Introduction}

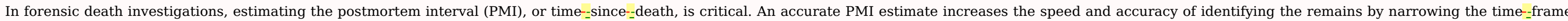

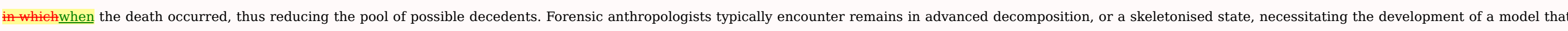

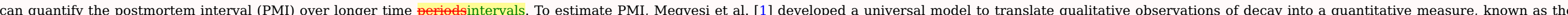




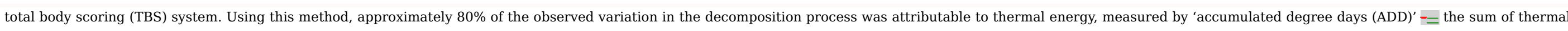

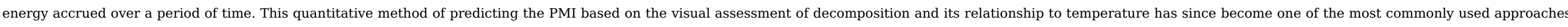

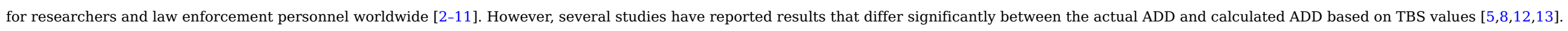

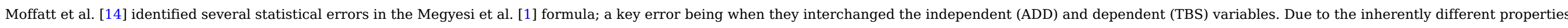

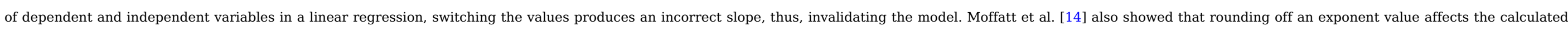

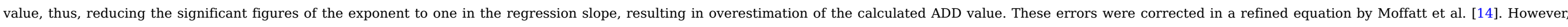
based on a review of the published literature, this new model has yet to be validated.

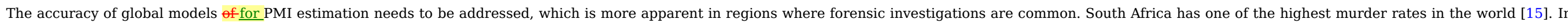

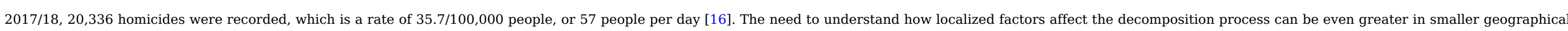
areas. The Western Cape province in South Africa includes the city of Cape Town, which is labelled as 'the murder capital of South Africa' with 3729 homicides (56.3/100,000 people) in 2017/18 [16].

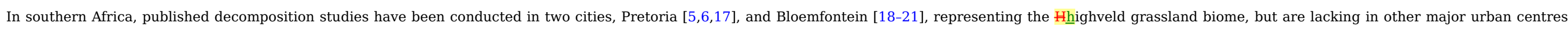

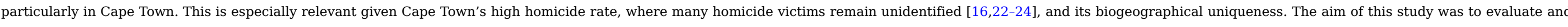

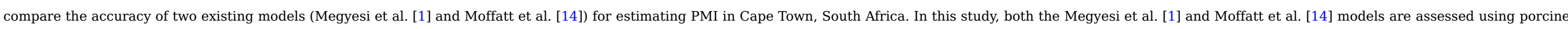

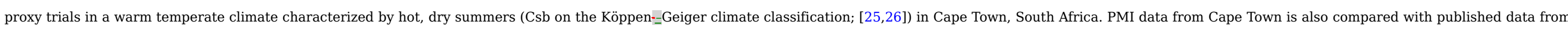

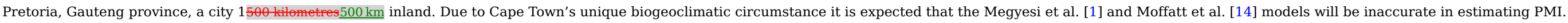

\section{Materials and methods}

\subsection{Study location}

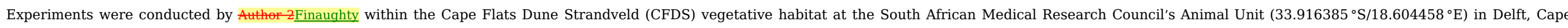

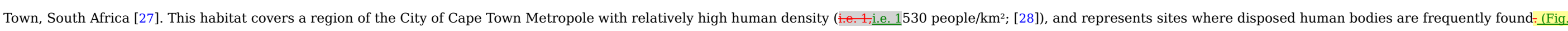
$\underline{1)}$.

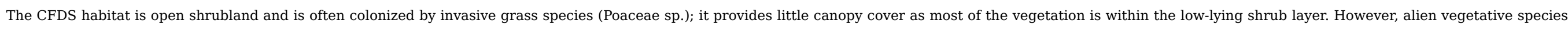

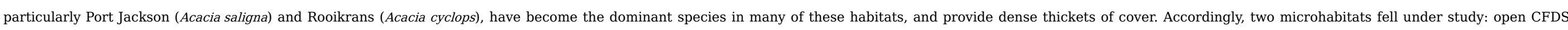
(CFDS/O) and closed (heavily vegetated) CFDS (CFDS/C).

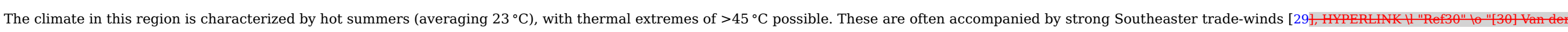

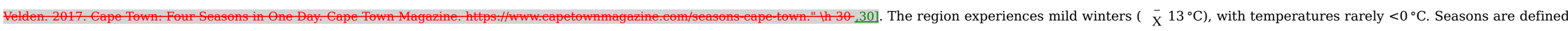

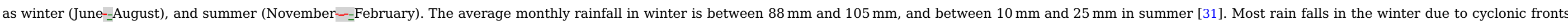
and the associated northwesterly winds.

\subsection{Pig carcasses}

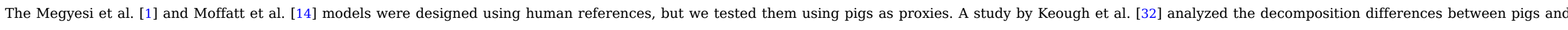

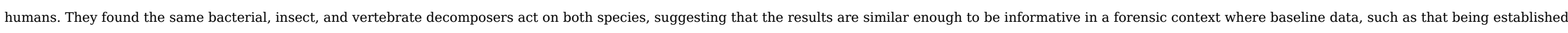
in the current study, are concerned. This has been affirmed in recent studies examining the differences in the rate and pattern of decomposition between simultaneously decomposing piq, human, and rabbit subjects [33,34].

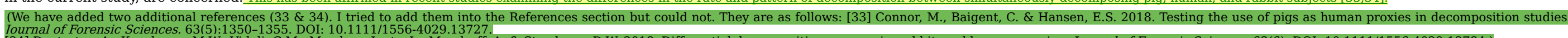

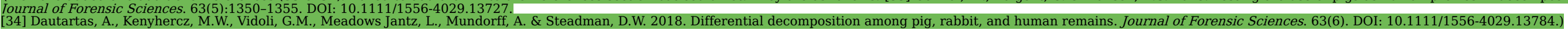

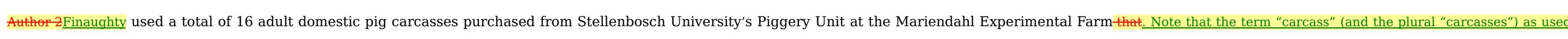

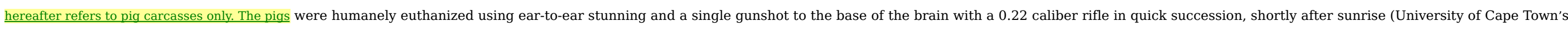




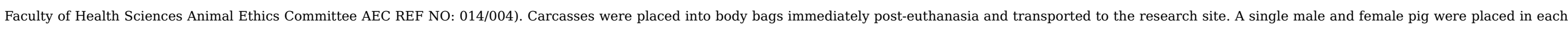

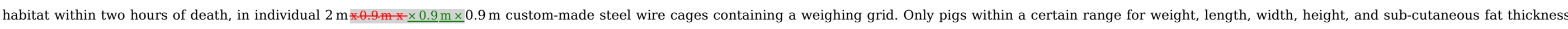

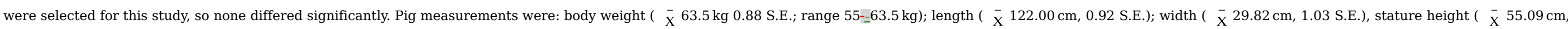
0.42 S.E.), and subcutaneous fat depth ( $\overline{\mathrm{X}} 6.38 \mathrm{~mm}, 0.20$ S.E.).

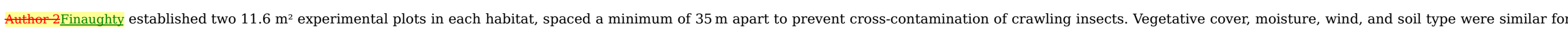

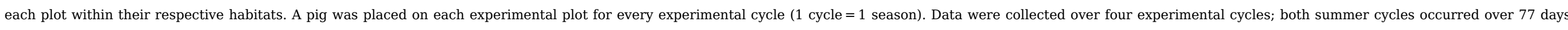
respectively, and the winter cycles each occurred over 142 days.

\subsection{Scoring decomposition}

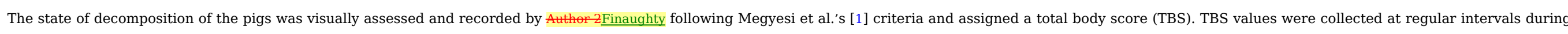

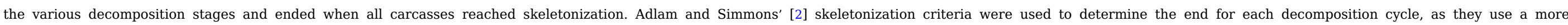
comprehensive definition.

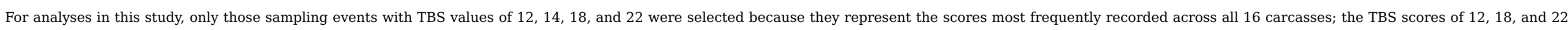
were recorded on 13 pigs, and TBS 14 was recorded on 12 pigs.

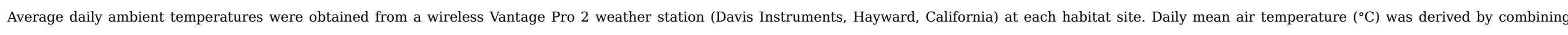

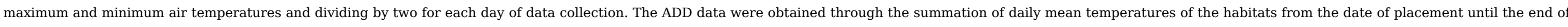
the cycle period.

Known ADD values ( $\mathrm{ADD}_{\text {actual }}$ ) to the estimated $\mathrm{ADD}$ values were calculated from the Megyesi et al. [1] model ( $\left.\mathrm{ADD}_{\mathrm{Meg}}\right)$ :

$\left(\mathrm{ADD}_{\mathrm{Meg}}=10^{(0.002 * \mathrm{TBS} * \mathrm{TBS}+1.81)} \pm 388.16\right) ;$

and the refined Moffatt et al. [14] model ( $\mathrm{ADD}_{\mathrm{Mof}}$ ):

$\left(\mathrm{ADD}_{\mathrm{Mof}}=10^{\left(\frac{\mathrm{TBS}+212}{125}\right)}\right)$

ADDactual values were calculated by summing the daily temperature averages for each carcass and $\mathrm{ADD}_{\mathrm{Meg}}$ and $\mathrm{ADD}$ Mof were calculated from specific TBS values $(12,14,18,22)$.

\subsection{Statistical analyses}

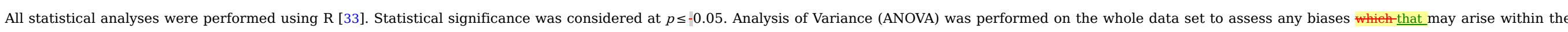

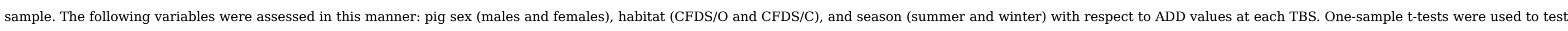

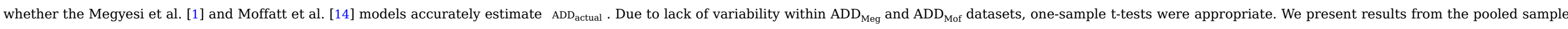
initially. Given the results of bias identification, data were separated and analysed within sub-groups (season and habitat).

\section{Results}

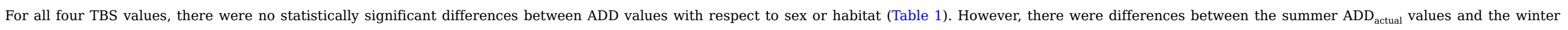

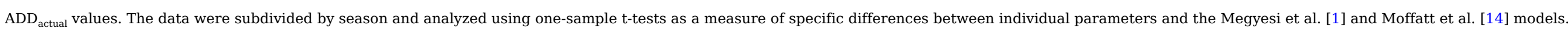

Table 1 Statistical ANOVA results for confounding variable within actual dataset by sex, habitat, and season for ADD values at TBS 12,14,18, and 22.

alt-text: Table 1 


\begin{tabular}{|l|l|}
\hline Sex & 1 \\
\hline Habitat & 1 \\
\hline Season & 1 \\
\hline Residuals & 10 \\
\hline Sex & 1 \\
\hline
\end{tabular}

\begin{tabular}{|l|}
\hline 2450 \\
\hline 205 \\
\hline 37698 \\
\hline 7084 \\
\hline 6035 \\
\hline
\end{tabular}

\begin{tabular}{|l|l|l|}
\hline 2450 & 3.4591 & 0.0925 \\
\hline 205 & 0.2900 & 0.6020 \\
\hline 37698 & 53.2146 & $<\mathbf{0 . 0 0 1}$ \\
\hline 708 & & 0.1743 \\
\hline 6035 & 2.1757 & 0.9140 \\
\hline 34 & 0.0123 & $<\mathbf{0 . 0 0 1}$ \\
\hline 104165 & 37.5525 & \\
\hline 2774 & & 0.5610 \\
\hline 3997 & 0.3616 & 0.0555 \\
\hline 51861 & 4.6917 & $<\mathbf{0 . 0 0 1}$ \\
\hline 1002923 & 90.7315 & \\
\hline 11054 & & 0.3945 \\
\hline 11994 & 0.7916 & 0.0544 \\
\hline 71895 & 4.7449 & $<\mathbf{0 . 0 0 1}$ \\
\hline 1671762 & 110.3316 & \\
\hline 15152 & & \\
\hline
\end{tabular}

\begin{tabular}{|l|l|l|}
\hline Habitat & 1 & 34
\end{tabular}

\begin{tabular}{l|l|l} 
Season & 1 & 34 \\
\hline
\end{tabular}

\begin{tabular}{l|l|l} 
Residuals & 9 & 24965
\end{tabular}

\begin{tabular}{l|l|l|}
\hline Habitat & 1 & 51861 \\
\hline Season & 1 & 1002923 \\
\hline
\end{tabular}

(20)

Items in bold were statistically significant $(p \leq 0.05)$. TBS $=$ total body score; $\mathrm{df}=$ degrees of freedom.

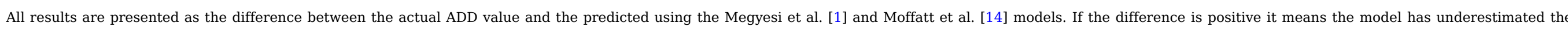
actual ADD, and if negative the actual ADD is overestimated by the model.

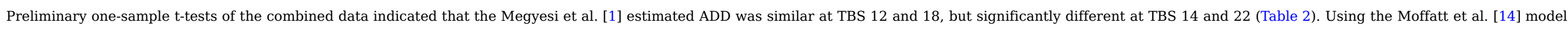
the four estimated ADD values were significantly different than the actual. Overall, the Megyesi et al. [1] ADD and the Moffatt et al. [14] ADD models underestimated the actual ADD (Fig. 1).

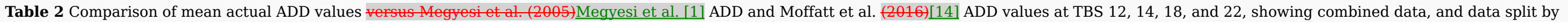
habitat and season. Percentage difference between actual ADD and Megyesi ADD, and actual ADD and Moffatt ADD are indicated.

\section{alt-text: Table 2}

TBS

$\mathrm{ADD}_{\text {actual }}$

$\mathrm{ADD}_{\mathrm{Meg}}$

\%diff

$\mathrm{ADD}_{\mathrm{Mof}}$

\%diff

\begin{tabular}{|c|c|c|c|c|c|c|c|c|c|c|}
\hline 12 & 140.73 & & & \multicolumn{2}{|l|}{125.31} & 11.59 & \multicolumn{2}{|l|}{92} & \multicolumn{2}{|l|}{41.88} \\
\hline 14 & 253.66 & & & \multicolumn{2}{|l|}{159.22} & 45.75 & \multicolumn{2}{|l|}{116} & \multicolumn{2}{|l|}{74.48} \\
\hline 18 & 456.57 & & & \multicolumn{2}{|l|}{287.08} & 45.58 & \multicolumn{2}{|l|}{201} & \multicolumn{2}{|l|}{77.73} \\
\hline 22 & 938.76 & & & \multicolumn{2}{|l|}{599.79} & 44.06 & \multicolumn{2}{|l|}{383} & \multicolumn{2}{|l|}{84.09} \\
\hline TBS & $\mathrm{ADD}_{\text {actual }}$ & $\mathrm{ADD}_{\text {Meg }}$ & $\%$ diff & $\mathrm{ADD}_{\mathrm{Mof}}$ & $\%$ diff & $\mathrm{ADD}_{\text {actual }}$ & $\mathrm{ADD}_{\text {Meg }}$ & \%diff & $\mathrm{ADD}_{\mathrm{Mof}}$ & \%diff \\
\hline \multicolumn{6}{|c|}{ CFDS Open Summer } & \multicolumn{5}{|c|}{ CFDS Closed Summer } \\
\hline
\end{tabular}




\begin{tabular}{|c|c|c|c|c|c|c|c|c|c|c|}
\hline 12 & 77.42 & 125.31 & 47.25 & 92 & 17.21 & 95.18 & 125.31 & 27.33 & 92 & 3.40 \\
\hline 14 & 133.61 & 159.22 & 17.49 & 116 & 14.11 & 165.3 & 159.22 & 3.75 & 116 & 35.05 \\
\hline 18 & 194.96 & 287.08 & 38.22 & 201 & 3.05 & 243.50 & 287.08 & 16.43 & 201 & 19.12 \\
\hline 22 & 631.07 & 599.79 & 5.08 & 383 & 48.93 & 553.08 & 599.79 & 8.10 & 383 & 36.34 \\
\hline TBS & $\mathrm{ADD}_{\text {actual }}$ & $\mathrm{ADD}_{\mathrm{Meg}}$ & \%diff & $\mathrm{ADD}_{\mathrm{Mof}}$ & \%diff & $\mathrm{ADD}_{\text {actual }}$ & $\mathrm{ADD}_{\mathrm{Meg}}$ & \%diff & $\mathrm{ADD}_{\mathrm{Mof}}$ & \%diff \\
\hline \multicolumn{6}{|c|}{ CFDS Open Winter } & \multicolumn{5}{|c|}{ CFDS Closed Winter } \\
\hline 12 & 191.55 & 125.31 & 41.81 & 92 & 70.21 & 197.03 & 125.31 & 44.50 & 92 & 72.68 \\
\hline 14 & 332.66 & 159.22 & 70.52 & 116 & 96.58 & 342.79 & 159.22 & 73.13 & 116 & 115.36 \\
\hline 18 & 823.59 & 287.08 & 96.61 & 201 & 121.53 & 671.86 & 287.08 & 80.25 & 201 & 107.89 \\
\hline 22 & 1294.93 & 599.79 & 73.38 & 383 & 108.70 & 1285.79 & 599.79 & 72.76 & 383 & 108.20 \\
\hline
\end{tabular}

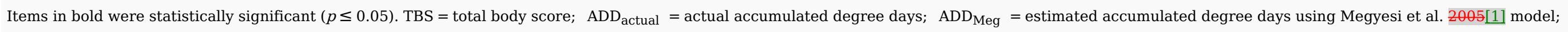
$\mathrm{ADD}_{\text {Mof }}=$ estimated accumulated degree days using Moffatt et al. 2016[14] model; CFDS = Cape Flats Dune Strandveld habitat.
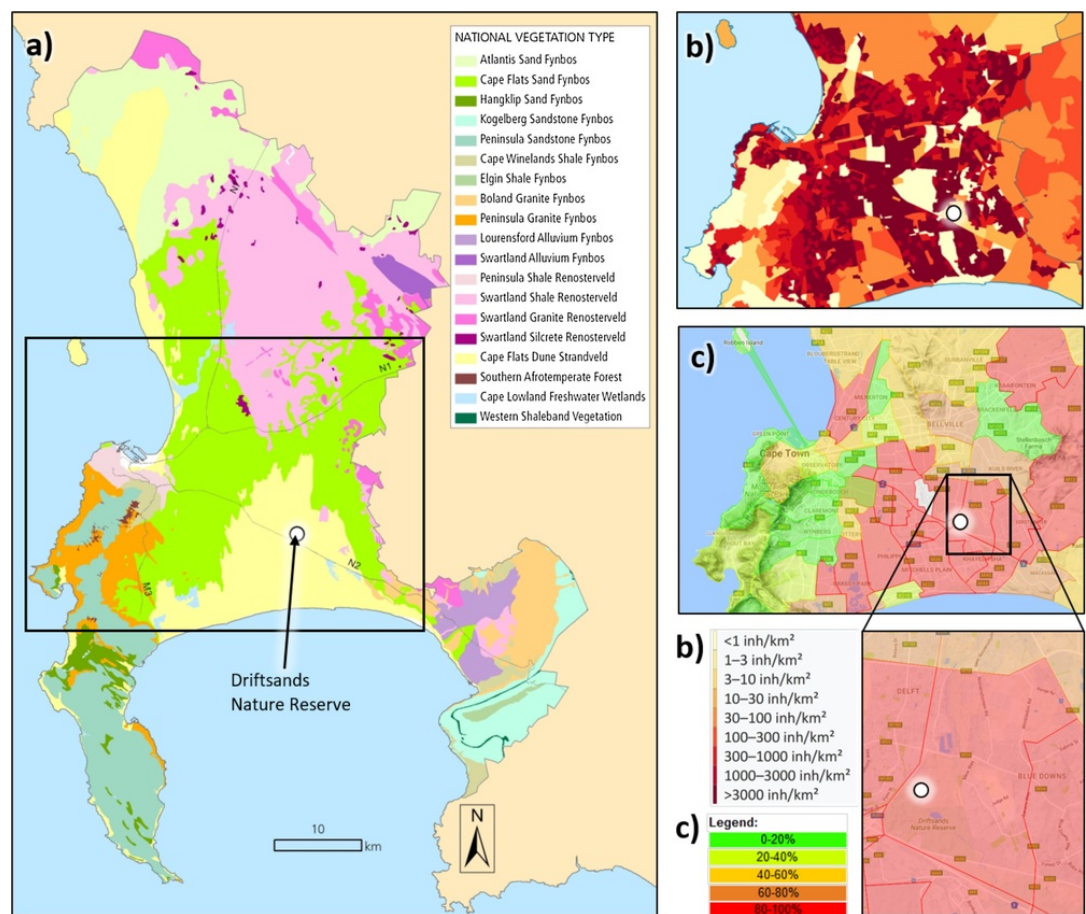

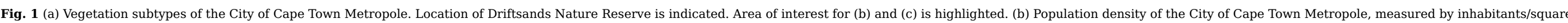

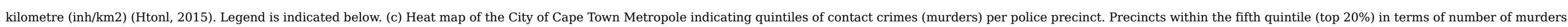

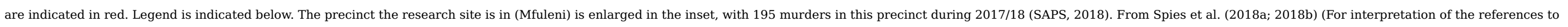
colour in this figure legend, the reader is referred to the web version of this article). 


\subsection{Separation by season \& habitat}

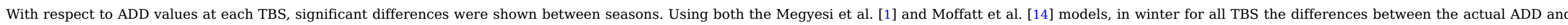

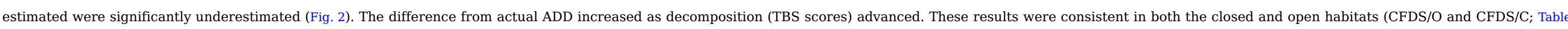

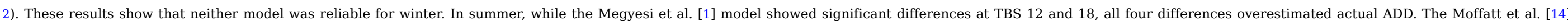

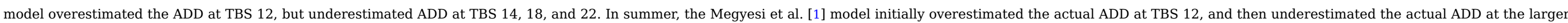
TBS scores.

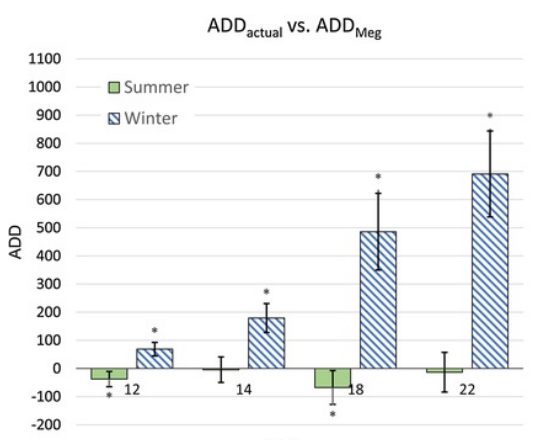

TBS

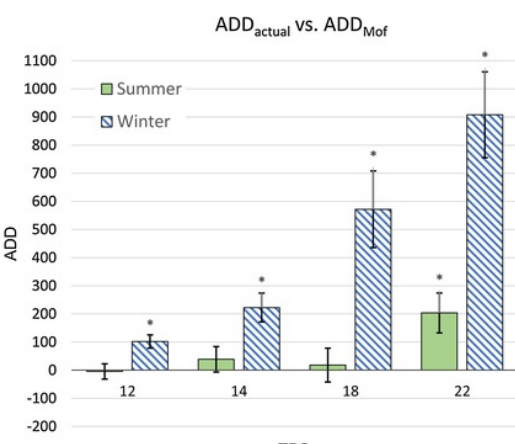

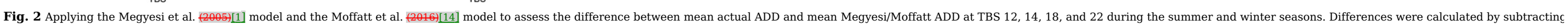
the mean Megyesi/Moffatt ADD from mean actual ADD. Significance $(p \leq 0.05)$ is denoted by the asterisk. alt-text: Fig. 2

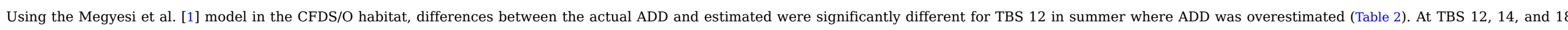

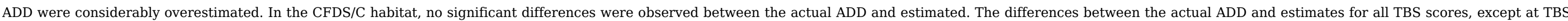
14, were negative. This is because the estimated ADD were larger than the actual ADD.

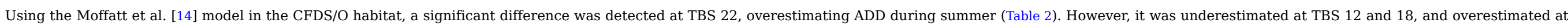
TBS 14 and 22. In the CFDS/C habitat, the difference between the actual and estimated ADD was significant for TBS 22, and this model consistently underestimated actual ADD.

\section{Discussion}

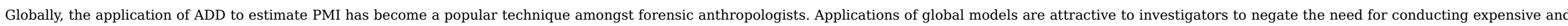

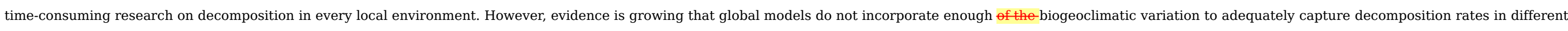
regions.

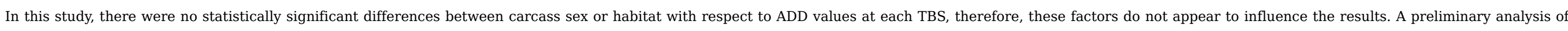

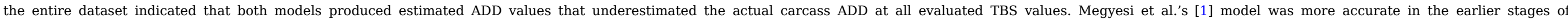

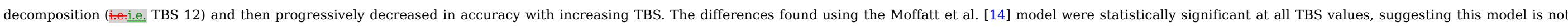
reliable in thisCape Town's environment.

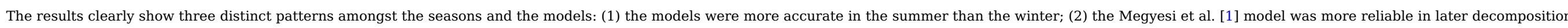
stages and (3) the Moffatt et al. [14] was more reliable in earlier decomposition stages. Each of these patterns are discussed below. 


\subsection{Summer vs. winter patterns W(Not sure what happened here. I tried to revise through the edit log but it would not work. Please double-check the words aren't duplicated (they appear so on my side; currently reads: "4.1 Summer vs. winter patterns Winter patterns"))inter patterns}

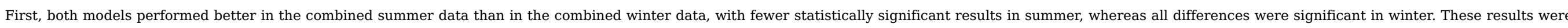

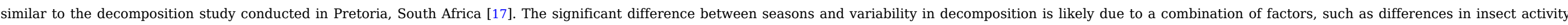

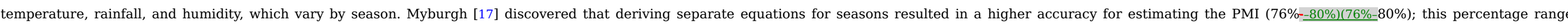

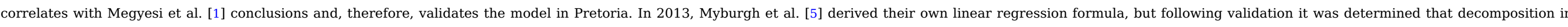

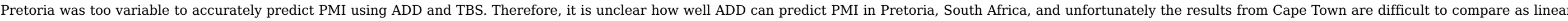
regression formulae have not yet been developed. This is an area of possible expansion on these preliminary results.

\subsection{Megyesi et al. [1] model}

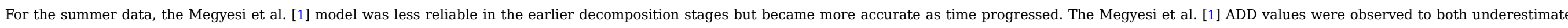

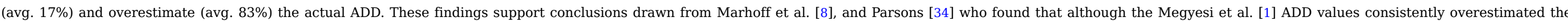
actual ADD, the model became increasingly more accurate towards the end of their studies. However, not all studies support this finding

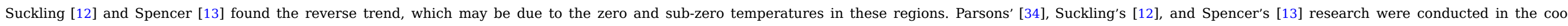

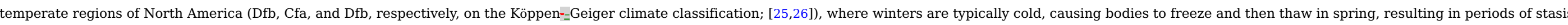

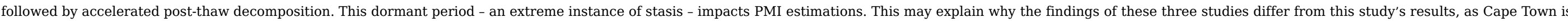

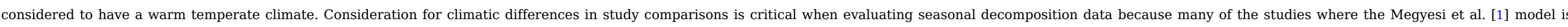

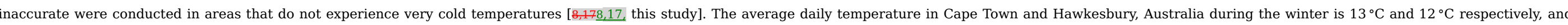

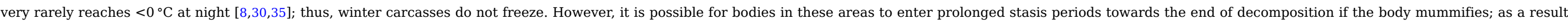

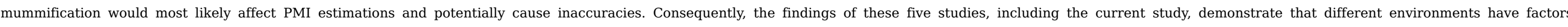
unaccounted for by the Megyesi et al. [1] model.

\subsection{Moffatt et al. [14] model}

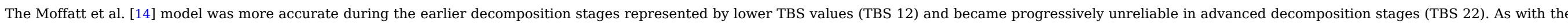

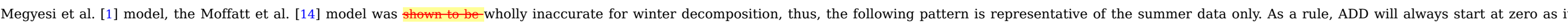

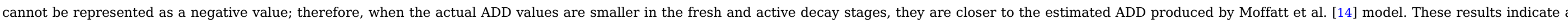

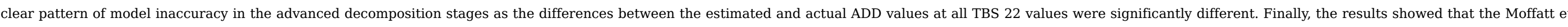

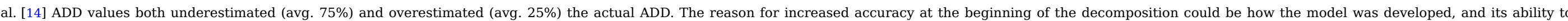
produce lower ADD values than those produced using the Megyesi et al. [1] model at the same TBS.

\subsection{Forensic implications}

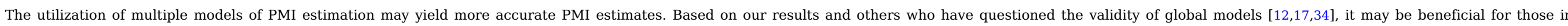

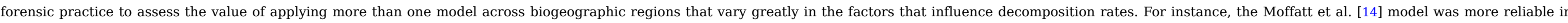

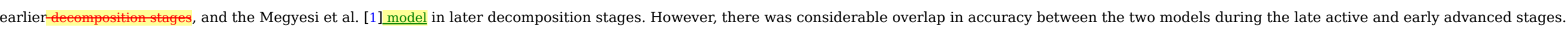

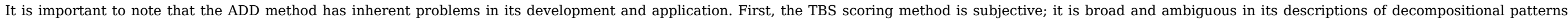

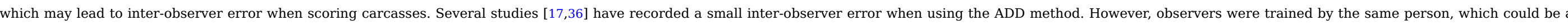

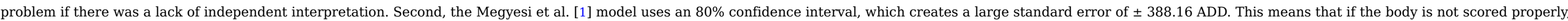




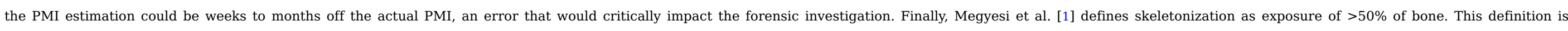

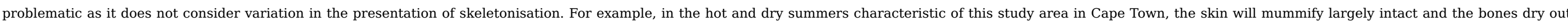
beneath the skin, meaning bone exposure may be minimal even though the carcass is essentially skeletonised. Therefore, using more inclusive criteria of skeletonization is better [4].

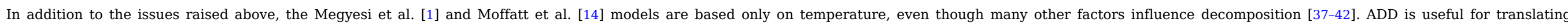

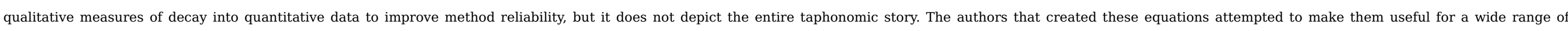

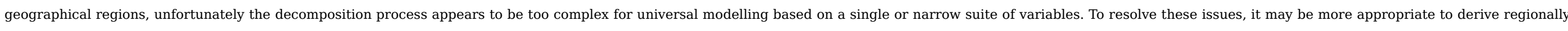

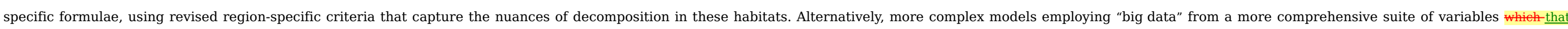
influence the rate and pattern of decay could be developed.

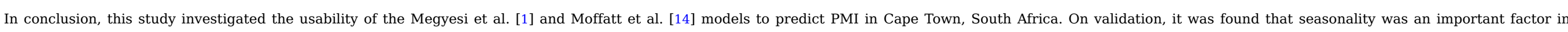

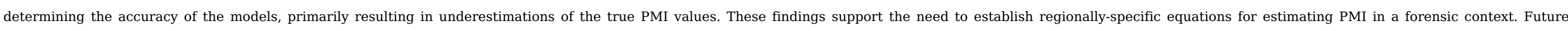
research should aim to establish regression formulae for winter and summer seasons in Cape Town, which can be validated using local forensic case work where bodies with known PMI are analyzed.

\section{Declarations of interest}

None.

\section{Grant declaration}

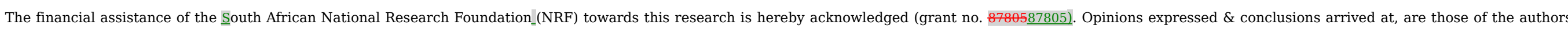
and are not necessarily to be attributed to the NRF.

\section{Acknowledgments}

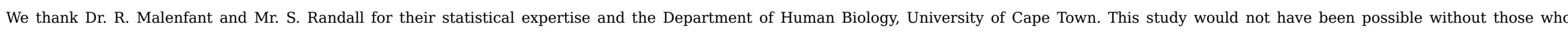

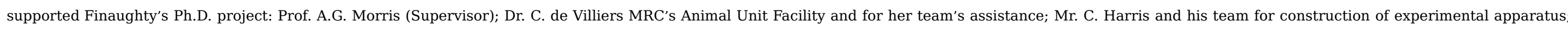

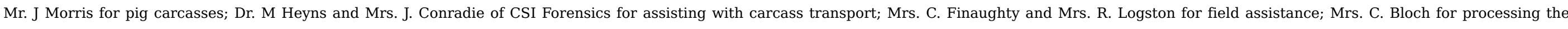
weather records.

\section{References}

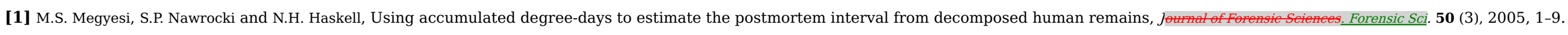

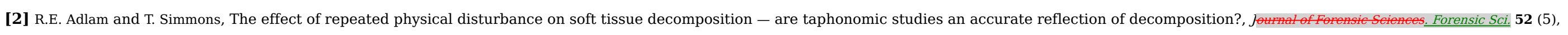
2007, 1007-1014, https://doi.org/10.1111/j.1556-4029.2007.00510.x.

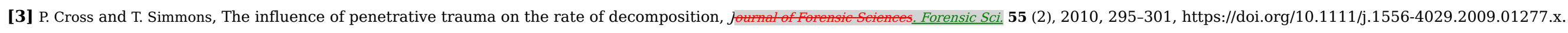

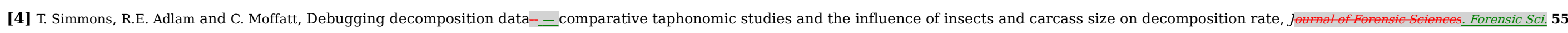
(1), 2010, 8-13, https://doi.org/10.1111/j.1556-4029.2009.01206.x.

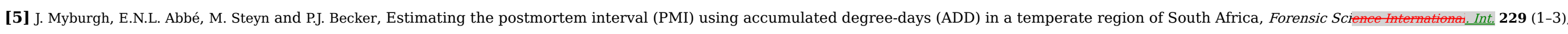
2013, e1-e6.

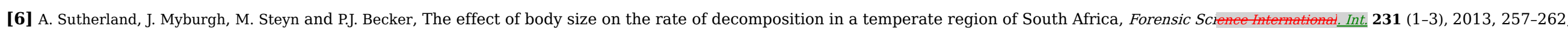
https://doi.org/10.1016/j.forsciint.2013.05.035. 


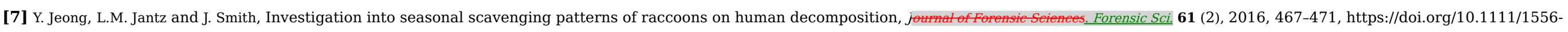
4029.12992 .

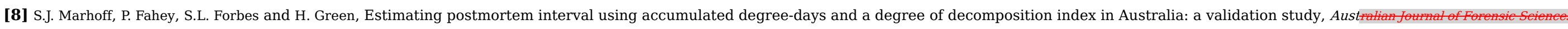
J. Forensic Sci. 48 (1), 2016, 24-36, https://doi.org/10.1080/00450618.2015.1021378.

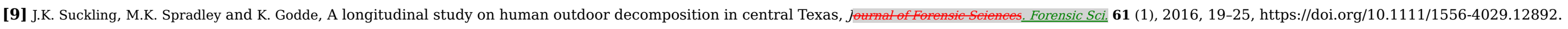

[10] D.L. Cockle and L.S. Bell, The environmental variables that impact human decomposition in terrestrially exposed contexts within Canada, Scienee and_Justice 57 (2), 2017, 107-117, https://doi.org/10.1016/j.scijus.2016.11.001.

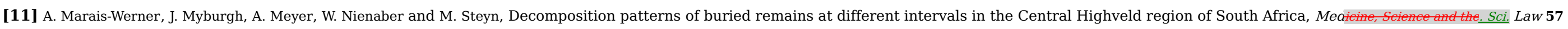
(3), 2017, 115-123, https://doi.org/10.1177/0025802417705263.

[12] J.K. Suckling, A tongitudinal study on the outdoor human decompesition sLongitudinal Study on the Outdoor Human Decomposition Sequence in Central Texas, -(May).(May)2011.

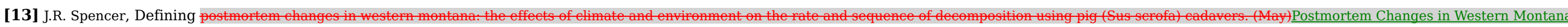
the Effects of Climate and Environment on the Rate and Sequence of Decomposition Using Pig (Sus Scrofa) Cadavers, (May)2013.

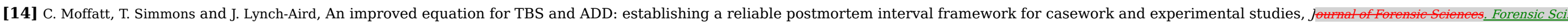
$\mathbf{6 1}$ (S1), 2016, S201-S207.

[15] H. Petr, 25 Countries with the Highest Murder Rates in the World. List 25: Geography \& Travel, https://list25.com/25-countries-with-the-highest-murder-rates-in-the-world/4/, 2018.

[16] South African Police Service, Crime Statistics 2017/2018, Available from:https://www.saps.gov.za/services/crimestats.php, 2018, [2018, September 12].(12 September 2018).

[17] J. Myburgh, Estimating the ostmortem interval using aceumulated dPostmortem Interval Using Accumulated Degree-days in a South African setting. (Oetober)Setting, (October)2010.

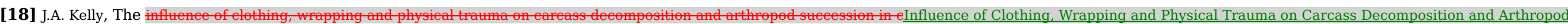
Succession in Central South Africa, 2006, University of the Free State.

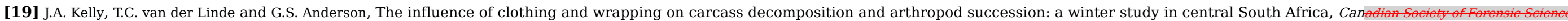
Fourna. Soc. Forensic Sci.J. 41 (3), 2008, 135-147, https://doi.org/10.1111/j.1556-4029.2009.01113.x.

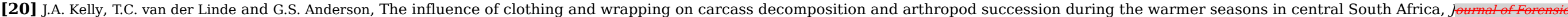
Sciences. Forensic Sci. $\mathbf{5 4}$ (5), 2009, 1105-1112, https://doi.org/10.1111/j.1556-4029.2009.01113.x.

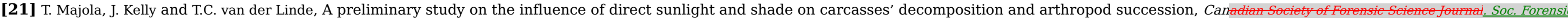
Sci.J. 46 (2), 2013, 93-102, https://doi.org/10.1080/00085030.2013.10757199.

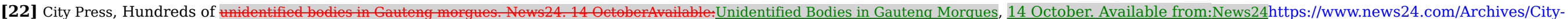
Press/Hundreds-of-unidentified-bodies-in-Gauteng-morgues-20150429, 2010, [2017, November 27].(27 November 2017).

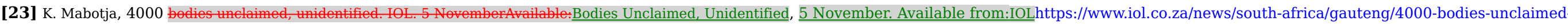
unidentified-1941249, 2015, £2017, November 271(27 November 2017).

[24] S. Wild, Long quest to understand these bodies without identities. Mail \& Guardian. 13 JanuaryAvailable:Quest to Understand These Bodies Without Identities, 13 January. Available from:Mail \& Guardianhttps://mg.co.za/article/2017-01-12-00-long-quest-to-understand-these-bodies-without-identities, 2017, [2017, November 27].(27 November 2017). 


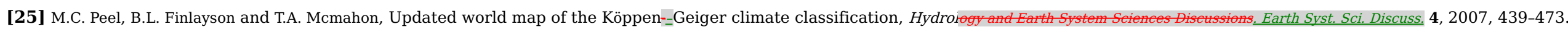

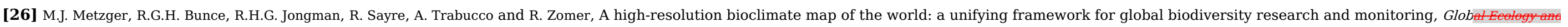
Biogregraphy. Ecol. Biogeogr. 22 (5), 2012, 1-9.

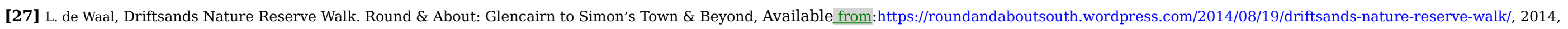
[2018, April 30](30 April 2018).

[28] Cape Town Population, World Population Review, Available from:http://worldpopulationreview.com/world-cities/cape-town-population/, 2018, [2018, May 9](9 May 2018).

[29] L. Mucina and M.C. Rutherford, (Eds.), The Vegetation of South Africa, Lesotho, and Swaziland, 2006, South African National Biodiversity Institute; Pretoria.

[30] Van der Velden, Cape Town: Four Seasons in One Day, Cape Town Magazinehttps://www.capetownmagazine.com/seasons-cape-town, 2017.

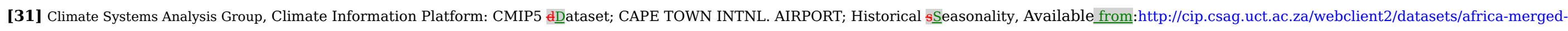
cmip5/\#nodes/seasonality-cmip5?folder_id=33\&extent=99919, 2018, [2018, October 16](16 October 2018).

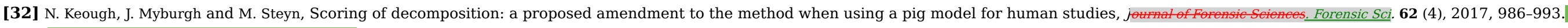
additional references have to go in here.)

[33ㅌ] R Core Team, R: A language and environment for statistical ea Language and Environment for Statistical Computing, R Foundation for Statistical Computinghttp://www.R-project.org/, 2013.

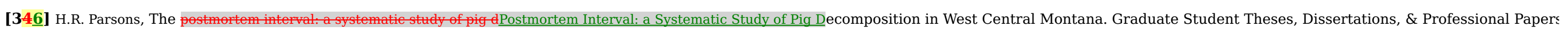
2009.

[357] Custom Weather, Climate \& Weather Averages in City of Hawkesbury, New South Wales, Australia, Time and Date.https://www.timeanddate.com/weather/@7302643/climate, 2018.

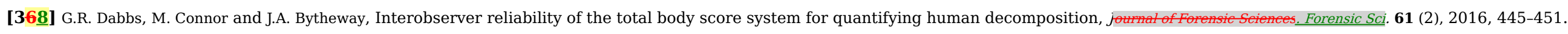

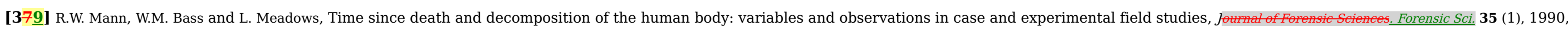
103-111, Available fom: http://www.ncbi.nlm.nih.gov/pubmed/2313251.

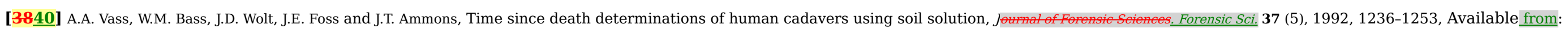
http://www.ncbi.nlm.nih.gov/pubmed/1402750.

[3941] C.P. Campobasso, G. Di Vella and F. Introna, Factors affecting decomposition and Diptera colonization, Forensic Scienee Internationat. Int. 120 (1-2), 2001, 18-27, Available from: http://www.ncbi.nlm.nih.gov/pubmed/11457604.

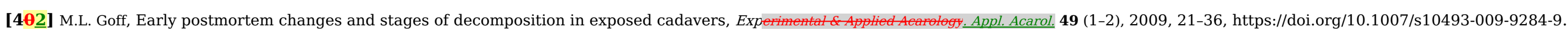

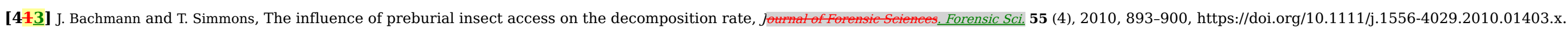

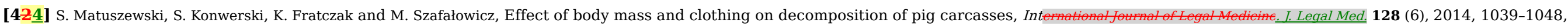
https://doi.org/10.1007/s00414-014-0965-5.

\section{Highlights}

- The accuracy of applying accumulated degree day models to various biogeoclimatic regions remains in question. 
